\title{
TRANSIENT DYNAMIC RESPONSE OF A NANOCOMPOSITE CONICAL SHELL WITH A RING STIFFENER UNDER THE ACTION OF AN IMPACT LOAD
}

\author{
${ }^{1}$ National Technical U niversity "K harkiv Polytechnic Institute" \\ $M$ inistry of Education and Science of $U$ kraine \\ 2 Kyrpychov St., Kharkiv 61002, U kraine; e-mail: omsroot@ kpi.kharkov.ua \\ ${ }^{2}$ A. Pidgorny Institute of Mechanical Engineering Problems \\ 2/10 Pozharsky St., Kharkiv 61046, U kraine; e-mail: admi@ ipmach.kharkov.ua
}

This work is devoted to the study of transient processes occurring in a nanocomposite shell with a ring stiffener under the action of an impact load. Nanocomposites are promising new materials for the aerospace industry. However, the analysis of dynamic processes in nanocomposite structures requires the development of new methods due to the anisotropic, functional-gradient nature of these materials. The problem is further complicated if a composed structure is to be analyzed.

This paper proposes a model of deformation of a functionally graded composite conical shell reinforced with carbon nanotubes with an isotropic ring stiffener. The deformation of the functionally graded nanocomposite conical shell is described by Reddy's high-order shear theory, and the deformation of the ring stiffener is described by the Euler-Bernoulli hypotheses. The Rayleigh-Ritz method is used to study the natural vibrations of the composite structure. The main variables are the displacements and angles of rotation of the conical shell.

A mathematical model of the transient response of the structure under the action of an impact load is obtained in the form of a linear dynamic system in generalized coordinates. To obtain this system, the prescribed form method is used.

Numerical studies of the free dynamics and transient response of a nanocomposite conical shell with an isotropic ring stiffener of rectangular section under the action of an impact load were carried out. The results of the numerical modeling of the transient process in the shell showed a close agreement with the results of finite element modeling in the ANSYS package.

The effect of the ring stiffener on the amplitudes of the transient response of the nanocomposite shell is investigated. It is shown that the ring-stiffener significantly reduces the amplitude of the transient response of the composite conical shell when it is subjected to an impact load. The proposed method and the conclusions drawn may be used in the aerospace industry in the design of nanocomposite units for multistage launch vehicles.

Keywords functionally graded nanocomposite, prescribed form method, linear dynamic system, transient response, compound shell.

1. Gibson R. F., Ayorinde E. O., Wen Y.-F. Vibrations of carbon nanotubes and their composites: A review. Composites Science and Technology. 2007. No. 67. Pp. 1-28

https://doi.org/10.1016/j.compscitech.2006.03.031

2. Qian D., Wagner G. J., Liu W. K., Yu M.-F., Ruoff R. S. Mechanics of carbon nanotubes. Appl. Mech. Rev. 2002. V. 55 (6). Pp. 495-533.

https://doi.org/10.1115/1.1490129

3. Raffi-Tabar H. Computational modelling of thermo-mechanical and transport properties of carbon nanotubes. Physics Reports. 2004. No. 390. Pp. 235-452.

https://doi.org/10.1016/j.physrep.2003.10.012

4. Coleman J. N., Khan U., Blau W. J., Gun'ko Yu. K. Small but strong: A review of the mechanical properties of carbon nanotube-polymer composites. Carbon. 2006. V. 44. Pp. 1624-1652. https://doi.org/10.1016/i.carbon.2006.02.038

5. Young R. J., Kinloch I. A., Gong L., Novoselov K. S. The mechanics of graphenenanocomposites: A review. Composites Science and Technology. 2012. V. 72. Pp. 1459-1476.

https://doi.org/10.1016/j.compscitech.2012.05.005

6. Njuguna J., Pielichowski K., Fan J. Polymer nanocomposites for aerospace applications. Advances in Polymer Nanocomposites. 2012. Pp. 472-539.

https://doi.org/10.1533/9780857096241.3.472

7. Pitchan M. K., Bhowmik S., Balachandran M., Abraham M. Process optimization of functionalized 
MWCNT/polyetherimide nanocomposites for aerospace application. 2017. V. 127. Pp. 193-203. https://doi.org/10.1016/j.matdes.2017.04.081

8. Allaoui A., Bai S., Cheng H. M., Bai J. B. Mechanical and electrical properties of a MWNT/epoxy composite. Composites Science and Technology. 2002. V. 62. Pp. 1993-1998. https://doi.org/10.1016/S0266-3538(02)00129-X

9. Ci L., Bai J. B. The reinforcement role of carbon nanotubes in epoxy composites with different matrix stiffness. Composites Science and Technology. 2006. V. 66. Pp. 599-603. https://doi.org/10.1016/j.compscitech.2005.05.020

10. Richard P., Prasse T., Cavaille J. Y., Chazeau L., Gauthier C., Duchet J. Reinforcement of rubbery epoxy by carbon nanofibres. Materials Science and Engineering. 2003. V. A352. Pp. 344-348. https://doi.org/10.1016/S0921-5093(02)00895-X

11. Nejati M., Asanjarani A., Dimitri R., Torna-bene F. Static and Free Vibration analysis of functionally graded conical shells reinforced by carbon nanotubes. International Journal of Mechanical Sciences. 2017. V. 130. Pp. 383-398.

https://doi.org/10.1016/j.ijmecsci.2017.06.024

12. Mehrabadi S. J., Aragh B. S. Stress analysis of functionally graded open cylindrical shell reinforced by agglomerated carbon nanotubes. Thin-Walled Structures. 2014. V. 80. Pp. 130-141. https://doi.org/10.1016/.tws.2014.02.016

13. Zhang L. W., Lei Z. X., Liew K. M., Yu J. L. Static and dynamic of carbon nanotube reinforced functionally graded cylindrical panels. Composite Structures. 2014. V. 111. Pp. 205-212. https://doi.org/10.1016/j.compstruct.2013.12.035

14. Song Z.G., Zhang L. W., Liew K. M. Vibration analysis of CNT-reinforced functionally graded composite cylindrical shells in thermal environments. International Journal of Mechanical Sciences. 2016. V. 115-116. Pp. 339-347. https://doi.org/10.1016/.i.jmecsci.2016.06.020

15. Sobhaniaragh B., Batra R. C., Mansur W. J., Peters F. C. Thermal response of ceramic matrix nanocomposite cylindrical shells using Eshelby-Mori-Tanaka homogenization scheme. Composites Part B: Engineering. 2017. V. 118. Pp. 41-53. https://doi.org/10.1016/j.compositesb.2017.02.032

16. Yaser K., Rossana D., Francesco T. Free vibration of FG-CNT reinforced composite skew cylindrical shells using the Chebyshev-Ritz formulation. Composites. Part B: Engineering. 2018. V. 147 Pp. 169-177.

https://doi.org/10.1016/j.compositesb.2018.04.028

17. Lei Z. X., Liew K. M., Yu J. L. Free vibration analysis of functionally graded carbon nanotubereinforced composite plates using the element-free kp-Ritz method in thermal environment. Composite Structures. 2013. V. 106. Pp. 128-138. https://doi.org/10.1016/j.compstruct.2013.06.003

18. Lei Z. X., Zhang L. W., Liew K. M. Elastodynamic analysis of carbon nanotube-reinforced functionally graded plates. International Journal of Mechanical Sciences. 2015. V. 99. Pp. 208-217. https://doi.org/10.1016/.j.jmecsci.2015.05.014

19. García-Macías E., Rodríguez-Tembleque L., Sáez A. Bending and free vibration analysis of functionally graded graphene vs. carbon nanotube reinforced composite plates. Composite Structures. 2018. V. 186. Pp. 123-138. https://doi.org/10.1016/j.compstruct.2017.11.076

20. Wang Q., Cui X., Qin B., Liang Q. Vibration analysis of the functionally graded carbon nanotube reinforced composite shallow shells with arbitrary boundary conditions. Composite Structures. 2017. V. 182. Pp. 364-379. https://doi.org/10.1016/j.compstruct.2017.09.043

21. Wang A., Chen H., Hao Y., Zhang W. Vibration and bending behavior of functionally graded 
nanocomposite doubly-curved shallow shells reinforced by graphene nanoplatelets. Results in Physics. 2018. V. 9. Pp. 550-559.

https://doi.org/10.1016/j.rinp.2018.02.062

22. Moradi-Dastjerdi R., Foroutan M., Pourasghar A. Dynamic analysis of functionally graded nanocomposite cylinders reinforced by carbon nanotube by a mesh-free method. Materials and Design. 2013. V. 44. Pp. 256-266.

https://doi.org/10.1016/j.matdes.2012.07.069

23. Amiro I. Ya., Zarutsky V. A. Shell Calculation Methods. Theory of Ribbed Shells. Kiev, 1980. (in Russian)

24. Bert C.W., Kim C.-D., Birman V. Vibration of composite-material cylindrical shells with ring and / or stringer stiffeners. Composite Structures. 1993. No. 25. Pp. 477-484.

https://doi.org/10.1016/0263-8223(93)90195-V

25. Jafari A.A., Bagheri M. Free vibration of rotating ring stiffened cylindrical shells with non-uniform stiffener distribution. Journal of Sound and Vibration. 2006. No. 296. Pp. 353-367.

https://doi.org/10.1016/j.jsv.2006.03.001

26. Kim Y.W., Lee Y.S. Transient analysis of ring-stiffened composite cylindrical shells with both edges clamped. Journal of Sound and Vibration. 2002. No. 252(1). Pp. 1-17 https://doi.org/10.1006/jsvi.2001.4020

27. Avramov K.V., Chernobryvko M., Uspensky B., Seitkazenova K.K., Myrzaliyev D. Self-sustained vibrations of functionally graded carbon nanotubes reinforced composite cylindrical shell in supersonic flow. Nonlinear Dynamics. 2019. No. 98(3). Pp. 1853-1876.

https://doi.org/10.1007/s11071-019-05292-z

28. Keleshteri M.M., Asadi H., Wang. Q. On the snap-through instability of postbuckled FG-CNTRC rectangular plates with integrated piezoelectric layers. Comp. Meth. Appl. Mech. Engine. 2018. No .331. Pp. 53-71.

https://doi.org/10.1016/j.cma.2017.11.015

29. Wang Q., Qin B., Shi D., Liang Q. A semi-analytical method for vibration analysis of functionally graded carbon nanotube reinforced composite doublycurved panels and shells of revolution. Comp. Struc. 2017. No. 174. Pp. 87-109.

https://doi.org/10.1016/j.compstruct.2017.04.038

30. Reddy J.N. A refined nonlinear theory of plates with transverse shear deformation. Int. J. of Sol. Struc. 1984. No. 20. Pp. 881-896.

https://doi.org/10.1016/0020-7683(84)90056-8

31. Amabili M., Reddy J.N. A new non-linear higher-order shear deformation theory for large-amplitude vibrations of laminated doubly curved shells. Int. J. of Non-Lin. Mech. 2010. No. 45. Pp. 409-418. https://doi.org/10.1016/j.ijnonlinmec.2009.12.013

32. Meirovitch L. Elements of vibration analysis. New York: McGraw-Hill Publishing Company, 1986. $495 \mathrm{pp}$.

33. Vlasov V.Z. Thin-Walled Elastic Beams. 2nd Edition. Washington: National Science Foundation, 1961. $493 \mathrm{pp}$.

34. Caresta M., Kessissoglou N. J.. Free vibrational characteristics of isotropic coupled cylindricalconical shells. Journal of Sound and Vibration. 2010. No. 329. Pp. 733-751.

https://doi.org/10.1016/j.jsv.2009.10.003

35. Chernobryvko M. V., Avramov K. V., Romanenko V. N., Batutina T. J., Tonkonogenko A. M. Free linear vibrations of thin axisymmetric parabolic shells. Meccanica. 2014. V. 49. No. 8. P. 2839-2845. https://doi.org/10.1007/s11012-014-0027-6

36. Shulzhenko N. G., Zaytsev B. F., Asaenik A. V. Numerical simulation of the dynamic response of 
structures to an impulse action. Aviatsionno-Kosmicheskaya Tekhnika i Tekhnologiya. 2014. No. 9. Pp. 6-11. (in Russian).

37. Shulzhenko N. G., Zaytsev B. F., Asaenok A. V., Klimenko D. V., Batutina T. Ya., Burchakov B. V. Dynamic impact interaction of space constructions adapters during separation. Space Sci.\&Technol. 2016. No. 22(2). Pp. 12-21. (in Russian). https://doi.org/10.15407/knit2016.02.012

Received on August 31, 2020 in final form on September 28, 2020 